\title{
Síndrome constitucional y alteración de las pruebas de función hepática en un paciente con fibrilación auricular
}

\author{
C. G. SUÁREZ, F. M UÑoz*, M. PORTILLO* \\ Residente de Medicina Interna de la Fundación J iménez Díaz. Madrid. *Residentes \\ de Medicina Familiar y Comunitaria. Centro de Salud Palacio de Segovia
}

\section{RESUMEN}

La alteración de las pruebas de función hepáti ca es un trastorno muy frecuente con el que el mé dico de familia debe enfrentarse en la práctica dia ria. Es muy importante descartar las causas más frecuentes con la realización y confirmación de pruebas básicas como son estudios bioquímicos, serologías y ecografía hepática. Pero también de bemos tener presentes otras causas como son el consumo de determinados fármacos y la presencia de otra patología concomitante. Para ello es fun damental la recogida exhaustiva de los datos de la historia clínica, así como la exploración del pa ciente.

En este caso presentamos un hipertiroidismo se cundario a amiodarona como responsable de la al teración hepática.

Palabras clave: Función hepática. Hipertiroi dismo. Amiodarona.
Asthenia, anorexia, weight loss and disturbances of the hepatic tests in a patient with atrial fibrilation

\section{INTRODUCCIÓN}

Discutimos el caso de un varón de 58 años con síndrome constitucional y alteración de las pruebas de función hepática que sigue tratamiento con amiodarona. Después de descartar otras causas más frecuentes compatibles con el cuadro se hace el diagnóstico de hipertiroidismo secundario a amiodarona.

El interés radica en resaltar la importancia de tener en cuenta la disfunción tiroidea en pacientes con hipertransaminasemia especialmente en casos

\begin{abstract}
General practitioners are frequently confronted with disturbances of the hepatic tests. It is therefore very important to be able to rule out the more usual alteration causes through basic tests such as bio chemical determinations, serological tests and ul trasound examination of the liver. Other causes such as the effects of certain drugs or the presence of a coincidental pathology must be taken into ac count as well. Having a full-made account of the patient's clinical history as well as performing the due physical explorations is therefore most useful to this purpose.

We show a case of hyperthyroidism related to amiodarone treatment as the responsible for the disturbances of the hepatic tests.
\end{abstract}

Key words: Hepatic tests. Hyperthyroidism. Amiodarone. que, como éste, siguen tratamiento con amiodarona. Aunque no es una circunstancia demasiado frecuente debemos conocer su diagnóstico y manejo, sobre todo para evitar otras pruebas innecesarias y agresivas ofreciendo al paciente una asistencia de calidad.

\section{CASO CLÍNICO}

Varón de 58 años con antecedentes de hipertensión arterial, hipercolesterolemia, hiperuricemia, fumador de 40 cigarrillos al día durante 33 años, 
bebedor de 25-30 unidades de alcohol a la semana; fibrilación auricular paroxística desde hace 3 años. La última revisión de su problema cardiológico, cinco meses antes, mostró que todas las pruebas complementarias se encontraban en los rangos de la normalidad (incluidas hormonas tiroideas). En el momento de la consulta seguía tratamiento con enalapril $20 \mathrm{mg} /$ día, clortalidona $25 \mathrm{mg} /$ día, atorvastatina $10 \mathrm{mg} /$ día, ácido acetilsalicílico 200 $\mathrm{mg} /$ día y amiodarona $400 \mathrm{mg} /$ día excepto los sábados y domingos.

El paciente comenzó tres semanas antes con un cuadro constitucional dominado por una importante astenia y pérdida de apetito con adelgazamiento de 8 kilogramos de peso (que suponen el $10 \%$ de su peso corporal). Además el paciente refería debilidad importante a nivel de las cinturas escapular y pélvica, más llamativa en la primera. Otro dato destacable es que aquejaba insomnio con nerviosismo. Una semana antes de la consulta presentó un episodio autolimitado de diarrea consistente en tres deposiciones líquidas al día sin productos patológicos ni dolor abdominal.

En la exploración física nos encontramos ante un paciente con buen estado general y buenas constantes (TA $120 / 70 \mathrm{~mm} \mathrm{Hg}, \mathrm{T}^{\mathrm{a}} 36,5^{\circ} \mathrm{C}$ y $78 \mathrm{lpm}$, peso $74 \mathrm{~kg}$ ), eupneico, bien nutrido y coloreado, no se palpaban adenopatías ni bocio. En la auscultación cardiopulmonar no había alteraciones. En el abdomen se palpaba el hígado a $1,5 \mathrm{~cm}$ del reborde costal, de consistencia blanda y sin otros hallazgos. Presentaba un discreto edema pretibial bilateral que dejaba fovea a la presión con pulsos periféricos conservados y simétricos.

Ante esta situación se solicitaron algunas pruebas complementarias cuyos resultados fueron los siguientes:

Hemograma: 4.640 leucocitos/ $\mathrm{mm}^{3}$ con fórmula normal, 4,17 millones de hematíes/ $\mathrm{mm}^{3}, \mathrm{Hb}$ $12,7 \mathrm{~g} / \mathrm{dl}$, Hto $36,3 \%$, VCM 87,1, HCM 30,5, 231.000 plaquetas $/ \mathrm{mm}^{3}$. VSG $23 \mathrm{~mm} / \mathrm{h}$.

Bioquímica: glucosa $83 \mathrm{mg} / \mathrm{dl}$, creatinina 0,7 $\mathrm{mg} / \mathrm{dl}$, urea $54 \mathrm{mg} / \mathrm{dl}$, ácido úrico 7,10 mg/dl, GGT $91 \mathrm{mg} / \mathrm{dl}$, GPT $143 \mathrm{mg} / \mathrm{dl}$, GOT $95 \mathrm{mg} / \mathrm{dl}$, fosfatasa alcalina $59 \mathrm{mg} / \mathrm{dl}$, bilirrubina total $0,50 \mathrm{mg} / \mathrm{dl}$, colesterol $140 \mathrm{mg} / \mathrm{dl}$, triglicéridos $221 \mathrm{mg} / \mathrm{dl}$, HDL colesterol $24 \mathrm{mg} / \mathrm{dl}$, colesterol VLDL $44 \mathrm{mg} / \mathrm{dl}$, colesterol LDL 72 mg/dl. Ferritina 1.172 ng/ml, hierro $47 \mu \mathrm{g} / \mathrm{dl}$, Transferrina $148 \mathrm{mg} / \mathrm{dl}$ e índice de saturación $25 \%$.

Radiografía de tórax: sin alteraciones significativas.

Serología de hepatitis: Ac HVC (-), Ac HB core (-), AcHBs (- ) y HAV Ig M (-)

Serología de CMV : Ig M (-) e Ig G (+).

Marcadores tumorales: alfa-fetoproteína, antígeno carcinoembrionario y antígeno prostático específico total negativos.

Ecografía abdominal: alteración difusa de la eco- genicidad hepática compatible con esteatosis sin lesiones ocupantes de espacio ni otros hallazgos.

Colonoscopia hasta el polo cecal sin alteraciones.

Hormonas tiroideas: TSH 0,01 mUI/1 (0,205,00), T4 libre 9,86 ng/dl $(0,85-2,1)$.

\section{DISCUSIÓN}

Nos encontramos ante un paciente con un síndrome constitucional y alteración de las pruebas de función hepática. Debemos tener en cuenta que un incremento de las transaminasas es un fenómeno inespecífico ${ }^{1}$ que puede ocurrir en gran número de situaciones patológicas y que no siempre traduce una enfermedad hepática. La valoración e interpretación de una hipertransaminasemia han de hacerse siempre dentro del contexto clínico del paciente y han de tenerse siempre presentes tanto la importancia cuantitativa de su elevación como el tiempo durante el que persiste la anomalía. La alta prevalencia de enolismo y hepatitis víricas en nuestro entorno hacen que estas etiologías deban descartarse de entrada ${ }^{2}$.

Iniciamos el estudio con la confirmación de las alteraciones bioquímicas ya que el primer paso siempre debe ser repetir la pruebas de laboratorio ${ }^{3}$ para descartar cualquier error. A continuación realizamos una anamnesis exhaustiva donde encontramos un antecedente de consumo de alcohol moderado (insuficiente para justificar una hepatopatía alcohólica), uso de atorvastatina que puede aumentar las transaminasas pero sin alteración del estado

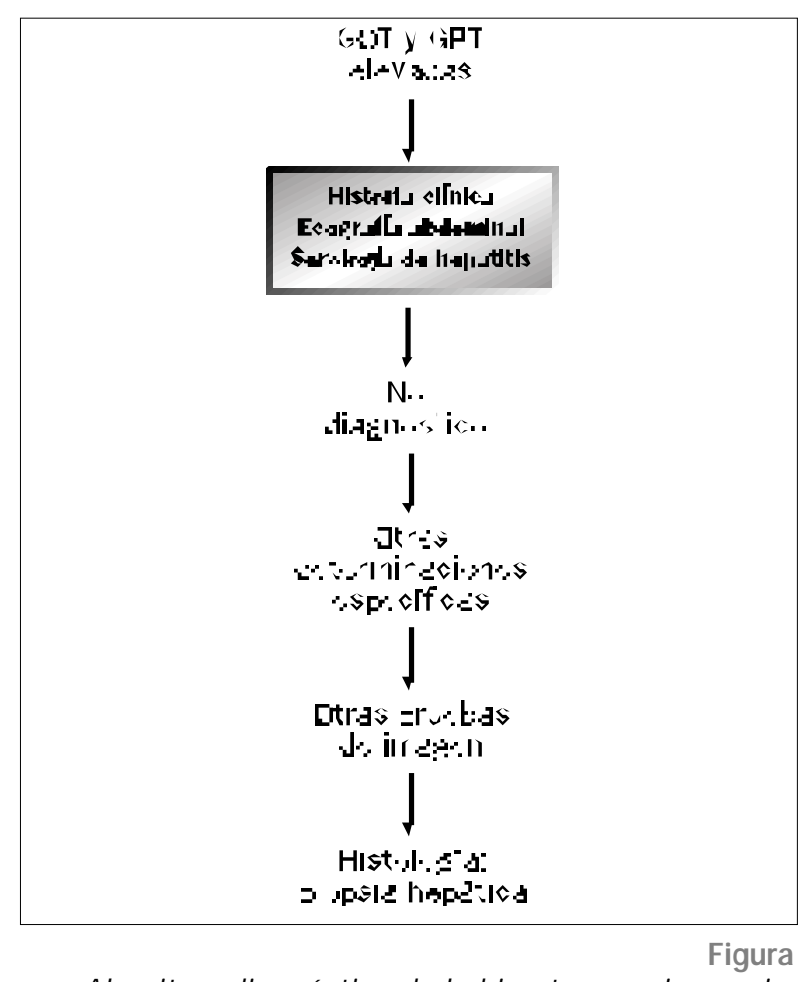

Algoritmo diagnóstico de la hipertransaminasemia. 
general, así como la ausencia de datos familiares de interés, prácticas de riesgo, transfusiones sanguíneas, etc. A continuación se le hizo una exploración física completa sin obtener datos reseñables salvo una discreta hepatomegalia. Con las serologías (primero de virus de la hepatitis y posteriormente de otros virus hepatotropos como son el citomegalovirus y el virus de Epstein-Barr) descartamos la posibilidad de hepatitis vírica y otros cuadros infecciosos parecían poco probables en el contexto del paciente. En la ecografía abdominal se aprecia una alteración ecogénica del hígado compatible con esteatosis hepática (no debemos olvidar que nuestro paciente presenta una hipercolesterolemia y consume alcohol) que, si bien podría justificar las alteraciones hepáticas, no parece justificar todo el cuadro de deterioro general y diarrea.
Dada la edad del paciente, la alteración del hábito intestinal y la elevación de la ferritina, otra opción diagnóstica que se nos plantea es la etiología tumoral, aunque con la ecografía habíamos descartado la presencia de lesiones ocupantes de espacio, y por ello decidimos la realización de la colonoscopia que descarta la existencia de una neoformación. Otra posibilidad sería la hemocromatosis pero no hay otros datos sugestivos (articulares, miocárdicos, gonadales, cutáneos, etc.) ni antecedentes familiares sugestivos y la saturación de la transferrina tampoco apoya el diagnóstico puesto que debería estar elevada.

Antes de recurrir a otros estudios más complejos y costosos, se decidió repetir la determinación de hormonas tiroideas, a pesar de tener un estudio rigurosamente normal cinco meses antes, porque el

Tabla

CAUSAS DE HIPERTRANSAMINASEMIA Y PRUEBAS DIAGNÓSTICAS

Causas hepáticas

Hepatopatía alcohólica

Fármacos hepatotóxicos

Hepatitis vírica

Hepatitis autoinmune

Hemocromatosis

Enfermedad de Wilson

Déficit de alfa-1-antitripsina

Cirrosis biliar primaria

Causas extrahepáticas

Enfermedad celiaca

Enfermedad de las vías biliares

Neoplasias con infiltración hepática

Enfermedades musculares

Hiper/ hipotiroidismo

Sarcoidosis

Ejercicio físico intenso

Porfiria cutánea tarda
Anamnesis, VCM aumentado, GGT aumentada

Anamnesis

Anti HVC, Ac HVB core, Ac HVBs, Ag HBs, Ac HVA IgM

ANA, ASM, anti-LKM

Hipergammaglobulinemia policlonal

Ferritina, índice de saturación de la ferritina, estudio genético

Cupremia, cupruria y ceruloplasmina en sangre

Proteinograma con descenso de alfa-globulinas, estudio genético (fenotipo ZZ)

AMA

Ac. antiendomisio y Ac. antigliadina

Ecografía abdominal, CPRE

Las pruebas dependerán de la localización del tumor primario

Aumento de enzimas musculares: GOT, LDH, CPK,...

Hormonas tiroideas (TSH,T4 libre)

ECA, radiografía de tórax, biopsia,...

Anamnesis

Determinación de porfirinas en sangre, heces y orina

VCM: Volumen Corpuscular Medio; ANA: Anticuerpos antinucleares; ASM: Anticuerpos anti-músculo liso; Anti-LKM: Anticuerpos contra los microsomas hepáticos y renales; AM A: Anticuerpos anti-mitocondriales.

CPRE: Colangiopancreatografía retrógrada endoscópica; ECA: Enzima convertidor de angiotensina. 
paciente continuaba en tratamiento con amiodarona. Los resultados fueron compatibles con el diagnóstico de hipertiroidismo en paciente tratado con amiodarona. La actitud que seguimos fue retirar el fármaco e iniciar el tratamiento con metimazol y betabloqueantes para controlar su arritmia.

La amiodarona es un antiarrítmico de clase III cuya estructura molecular es muy similar a la triiodotironina. Contiene dos átomos de yodo, lo que le confiere un $37,2 \%$ del peso en yodo. Esto supone que con una dosis de mantenimiento de 200 a 600 $\mathrm{mg}$ se aporten 75 a $225 \mathrm{mg} /$ día de yodo ${ }^{4}$. Este exceso de yodo puede tener consecuencias sobre la fisiología tiroidea. Por otra parte debemos tener en cuenta que es una molécula muy lipofílica, lo que le confiere una vida media muy prolongada (22-55 días), pudiendo detectarse en plasma hasta 9 meses después de retirado el fármaco.

El tratamiento con amiodarona provoca una serie de cambios en las hormonas tiroideas que consisten en un aumento de la T4 total y libre y de rT3, una disminución de la T3 total, junto con un aumento inicial y precoz de TSH que tiende a normalizarse, aunque en ocasiones persiste aumentado tras tratamientos crónicos. Estos cambios se producen en más del $50 \%$ de los pacientes en tratamiento con amiodarona, sin ninguna relevancia clínica (estado eutiroideo).

La incidencia de disfunción tiroidea oscila entre un 2 y un $24 \%$. En nuestro medio la incidencia de tirotoxicosis por amiodarona se encuentra en torno al $1,5 \%$ y es más frecuente en zonas con déficit de yodo. Se han descrito dos mecanismos fisiopatológicos diferentes ${ }^{6}$ :

Tipo I: se produce sobre tiroides con patología subyacente con una alteración en el mecanismo autorregulador de la captación de yodo que llevaría a un aumento de la misma con el consiguiente aumento en la síntesis de hormona tiroidea.

Tipo II: se produce sobre glándulas aparentemente normales, sin bocio y con anticuerpos antiroideos y antirreceptor de TSH negativos. El mecanismo sería una citotoxicidad inducida por la amiodarona o el yodo.
La manifestación clínica más frecuente en estos casos suele ser un empeoramiento de la enfermedad cardiaca subyacente, aunque pueden existir los síntomas clásicos de hipertiroidismo.

En ocasiones el curso de la enfermedad es autolimitado, resolviéndose la situación tras la retirada de la amiodarona y sin tratamiento adicional, aunque generalmente tras largos periodos de tiempo. La resolución del cuadro es más precoz cuando no existe patología subyacente. El tratamiento incluye tionamidas que pueden asociarse con perclorato potásico para conseguir una rápida descarga del yodo intratiroideo aumentando su eficacia, corticoides, plasmaféresis en casos de crisis tireotóxicas que ponen en peligro la vida del paciente, litio y tratamiento quirúrgico.

Bartalena ha propuesto un tratamiento específico según el tipo. En el tipo I, en el que existe una síntesis de hormona tiroidea aumentada, el tratamiento de elección serían los antitiroideos junto a perclorato potásico, mientras que los corticoides tendrían un papel limitado. Sin embargo, en el tipo II, en el que el mecanismo fundamental es una destrucción tisular, el tratamiento de elección serían los corticoides en dosis altas, por su efecto antiinflamatorio y estabilizador de membranas.

La cirugía está indicada en casos graves con arritmias potencialmente letales para el paciente en los que se requiera una solución rápida, cuando no se pueda retirar la amiodarona y cuando se haya demostrado una resistencia al tratamiento médico. Debe practicarse una tiroidectomía total o casi total sobre todo en aquellos pacientes que vayan a seguir el tratamiento con amiodarona.

\section{CORRESPONDENCIA:}

Carmen G. Suárez Álvarez

Unidad Hospitalización a Domicilio

Fundación Jiménez Díaz

Avda. Reyes Católicos, 2

28040 Madrid

\section{Bibliografía}

1. Moitinho E, Bruguera M. Protocolo diagnóstico de las elevaciones agudas de las transaminasas. Medicine 1996; 7 (9): 379-81.

2. Sort P. Protocolo diagnóstico ante la elevación crónica de las transaminasas. Medicine 1996; 7 (9): 382-5.

3. Pratt DS, Kaplan MM. Primary Care: Evaluation of abnormal liver-enzyme results in asymptomatic patients. N Eng J Med 2000; 342 (17): 1266-71.
4. Harjai KJ, Licata AA. Efects of amiodarone on Thyroid function. Ann Intern Med 1997; 126: 63-73.

5. Lahera M, De Luis A, Hervilla S, Maldonado G, Zurita P. Amiodarona y tiroides. Rev Clin Española 1999; 199 (4): 227-32.

6. Bartalena L, Brogioni S, Grasso L, Bogazzi F, Burelli A, Martino E. Treatment of amiodarone-induced thyrotoxicosis, a difficult challenge: results of a prospective study. J Clin Endocrinol Metab 1996; 81: 2930-3. 